\title{
Stem cell treatment improves post stroke neurological outcomes: a comparative study in male and female rats
}

\author{
Koteswara Rao Nalamolu, ${ }^{1,2}$ Bharath Chelluboina, ${ }^{1,3}$ Casimir A Fornal, ${ }^{1}$ \\ Siva Reddy Challa, ${ }^{1}$ David M Pinson, ${ }^{4}$ David Z Wang, ${ }^{5,6}$ Jeffrey D Klopfenstein, ${ }^{1,7,8}$ \\ Krishna Kumar Veeravalli (D) 1,5,7,9
}

To cite: Nalamolu KR, Chelluboina B, Fornal CA, et al. Stem cell treatment improves post stroke neurological outcomes: a comparative study in male and female rats. Stroke \& Vascular Neurology 2021;0. doi:10.1136/svn-2020-000834

- Additional material is published online only. To view, please visit the journal online (http://dx.doi.org/10.1136/svn2020-000834).

Received 26 December 2020 Revised 12 February 2021 Accepted 18 February 2021
A) Check for updates

(c) Author(s) (or their employer(s)) 2021. Re-use permitted under CC BY-NC. No commercial re-use. See rights and permissions. Published by BMJ.

For numbered affiliations see end of article.

Correspondence to Dr Krishna Kumar Veeravalli; krishnav@uic.edu

\section{ABSTRACT}

Background and purpose The therapeutic potential of different stem cells for ischaemic stroke treatment is intriguing and somewhat controversial. Recent results from our laboratory have demonstrated the potential benefits of human umbilical cord blood-derived mesenchymal stem cells (MSC) in a rodent stroke model. We hypothesised that MSC treatment would effectively promote the recovery of sensory and motor function in both males and females, despite any apparent sex differences in post stroke brain injury.

Methods Transient focal cerebral ischaemia was induced in adult Sprague-Dawley rats by occlusion of the middle cerebral artery. Following the procedure, male and female rats of the untreated group were euthanised 1 day after reperfusion and their brains were used to estimate the resulting infarct volume and tissue swelling. Additional groups of stroke-induced male and female rats were treated with MSC or vehicle and were subsequently subjected to a battery of standard neurological/ neurobehavioral tests (Modified Neurological Severity Score assessment, adhesive tape removal, beam walk and rotarod). The tests were administered at regular intervals (at days 1, 3, 5, 7 and 14) after reperfusion to determine the time course of neurological and functional recovery after stroke.

Results The infarct volume and extent of swelling of the ischaemic brain were similar in males and females. Despite similar pathological stroke lesions, the clinical manifestations of stroke were more pronounced in males than females, as indicated by the neurological scores and other tests. MSC treatment significantly improved the recovery of sensory and motor function in both sexes, and it demonstrated efficacy in both moderate stroke (females) and severe stroke (males).

Conclusions Despite sex differences in the severity of post stroke outcomes, MSC treatment promoted the recovery of sensory and motor function in male and female rats, suggesting that it may be a promising treatment for stroke.

\section{INTRODUCTION}

Pharmacological treatments that facilitate neurological recovery after ischaemic stroke remain an unmet clinical need. As an alternative approach, therapy with cells offers a potential regenerative treatment to restore post stroke deficits and disabilities. ${ }^{1}$ Cell-based therapies could replace the dead or damaged cells in the ischaemic core and rescue the dying tissue in the penumbra. ${ }^{23}$ Treatment with stem cells promotes vasculogenesis, neurogenesis and synaptogenesis, reduces scar thickness, enhances autophagy and inhibits inflammation-induced brain damage, all of which are likely to be beneficial in stroke recovery. ${ }^{4-9}$ Following systemic administration, stem cell grafts preferentially migrate to the spleen and abrogate chronic inflammation after stroke. ${ }^{10}$ Together, these protective mechanisms could contribute to the restoration of post stroke neurological function.

The therapeutic potential of different stem cells for ischaemic stroke treatment is intriguing and somewhat controversial. ${ }^{11}$ Recent results from our laboratory employing a rodent model of ischaemic stroke have demonstrated that treatment with human umbilical cord blood-derived mesenchymal stem cells (MSC) reduces apoptosis and post stroke brain damage, downregulates DNA damage-inducing genes, upregulates DNA repair genes and attenuates the induction of matrix metalloproteinases in the ischaemic brain. ${ }^{12-14}$ The administration of MSC to rabbits after ischaemic stroke was also shown to suppress the inflammatory responses and neuronal apoptosis. ${ }^{15}$ More recently, we have reported that exosomes derived from these MSC under the appropriate experimental conditions improve the functional recovery of stroke-induced rats. ${ }^{16}$ While the aforementioned studies reporting enhanced neurological recovery after human umbilical cord blood-derived MSC treatment in both rodent and non-rodent models are encouraging, the limited neurological testing conducted in 
these studies precluded any strong conclusions regarding the efficacy of these treatments on sensory and motor function. ${ }^{17}{ }^{18}$ Furthermore, another shortcoming of these studies is that they were not conducted in both sexes as recommended by the Stroke Treatment Academic Industry Roundtable. ${ }^{19}$

It is well known that stroke incidence in young-aged to middle-aged adults is lower in women than men. However, this trend reverses later in life, especially when women enter the postmenopausal stage.$^{20}$ Similarly, young female animals appear to be more resistant to ischaemic brain damage than age-matched males. ${ }^{21}{ }^{22}$ Studies in ovariectomised rodents have revealed that oestrogens provide significant neuroprotection against ischaemic stroke in females. ${ }^{21} 23$ Other studies have provided additional evidence for reduced infarct size and neuroprotective action of oestrogen. ${ }^{24-26}$ However, oestrogen increased infarct volume in aged female rodents. ${ }^{27}{ }^{28}$ In summary, these studies suggest that oestrogen protects the younger brain but exacerbates the damage in the ageing brain, after ischaemic stroke. Thus, oestrogen may not be the only causative factor for sexual dimorphism in post stroke brain injury. This is further supported by the divergent cell death pathways in males and females after ischaemic stroke. $^{20}$

The purpose of the present study was twofold: (1) to investigate the efficacy of MSC treatment on post stroke sensory and motor function, using a battery of neurobehavioral tests and multiple timepoint assessments over an extended period in both male and female rats, and (2) to determine whether the efficacy of MSC treatment is similar or different between the two sexes. We hypothesised that MSC treatment, by its very nature, would be effective in promoting the recovery of sensory and motor function in both males and females despite any apparent sex differences in post stroke brain injury.

\section{METHODS}

A total of 50 young adult male and 50 young adult female Sprague-Dawley rats were used in this study. Of this number, 20 rats (10 males and 10 females) were used to evaluate possible sex differences in brain infarct size or swelling after ischaemic stroke. These animals were assigned to untreated ischaemic cohorts designated respectively as Untreated Males and Untreated Females. The remaining 80 animals were used to evaluate the effects of MSC treatment on post stroke deficits. The animals were randomly assigned by sex to one of the two treatment cohorts resulting in four groups, each consisting of 20 animals. The groups were vehicletreated males (Vehicle Males), vehicle-treated females (Vehicle Females), MSC-treated males (MSC Males) and MSC-treated females (MSC Females). Rats from all the groups were subjected to a 2-hour middle cerebral artery occlusion (MCAO) followed by reperfusion as previously described by our group. ${ }^{14}$ For stem cell treatment, approximately 0.25 million human umbilical cord blood-derived MSC suspended in sterile PBS were administered intravenously (tail vein) to the appropriate groups of strokeinduced rats at 1 day after reperfusion. PBS was administered to rats in the vehicle-treated groups. A schematic diagram of the experimental design is shown in figure 1 .

Brain injury was assessed in the untreated rats by performing triphenyltetrazolium chloride (TTC) staining at 1 day after reperfusion. The percent infarct volume was quantified by using the formula: Infarct volume $(\%)=[$ (volume of contralateral hemisphere-volume of non-ischaemic ipsilateral hemisphere)/volume of contralateral hemisphere $] \times 100$. This formula takes into account the possible interference of brain oedema/swelling on infarct volume. Swelling of the ipsilateral hemisphere was calculated by using the formula: Swelling $(\%)=[$ (volume

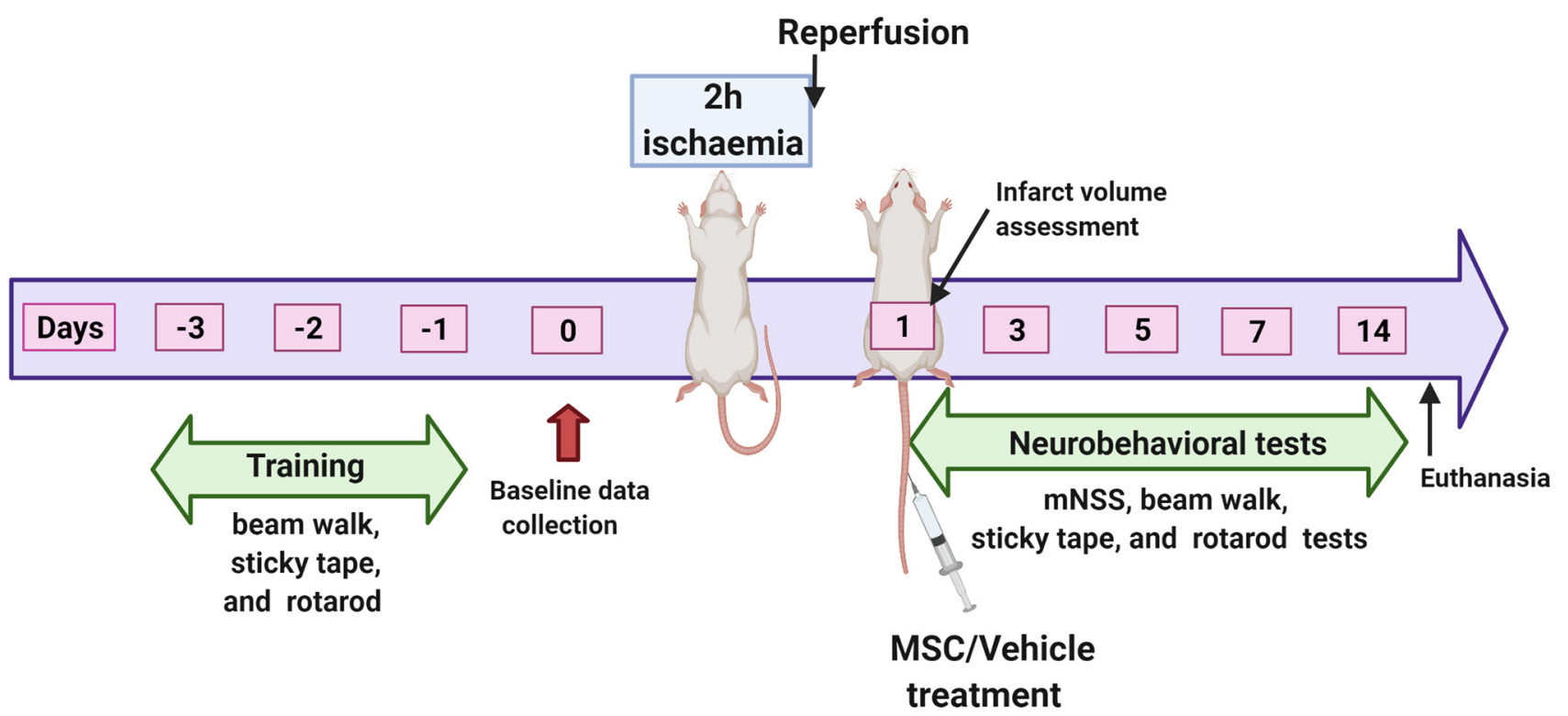

Figure 1 Schematic representation of the experimental design. mNSS, Modified Neurological Severity Score; MSC, mesenchymal stem cells. The figure was created with BioRender.com under a paid subscription. 

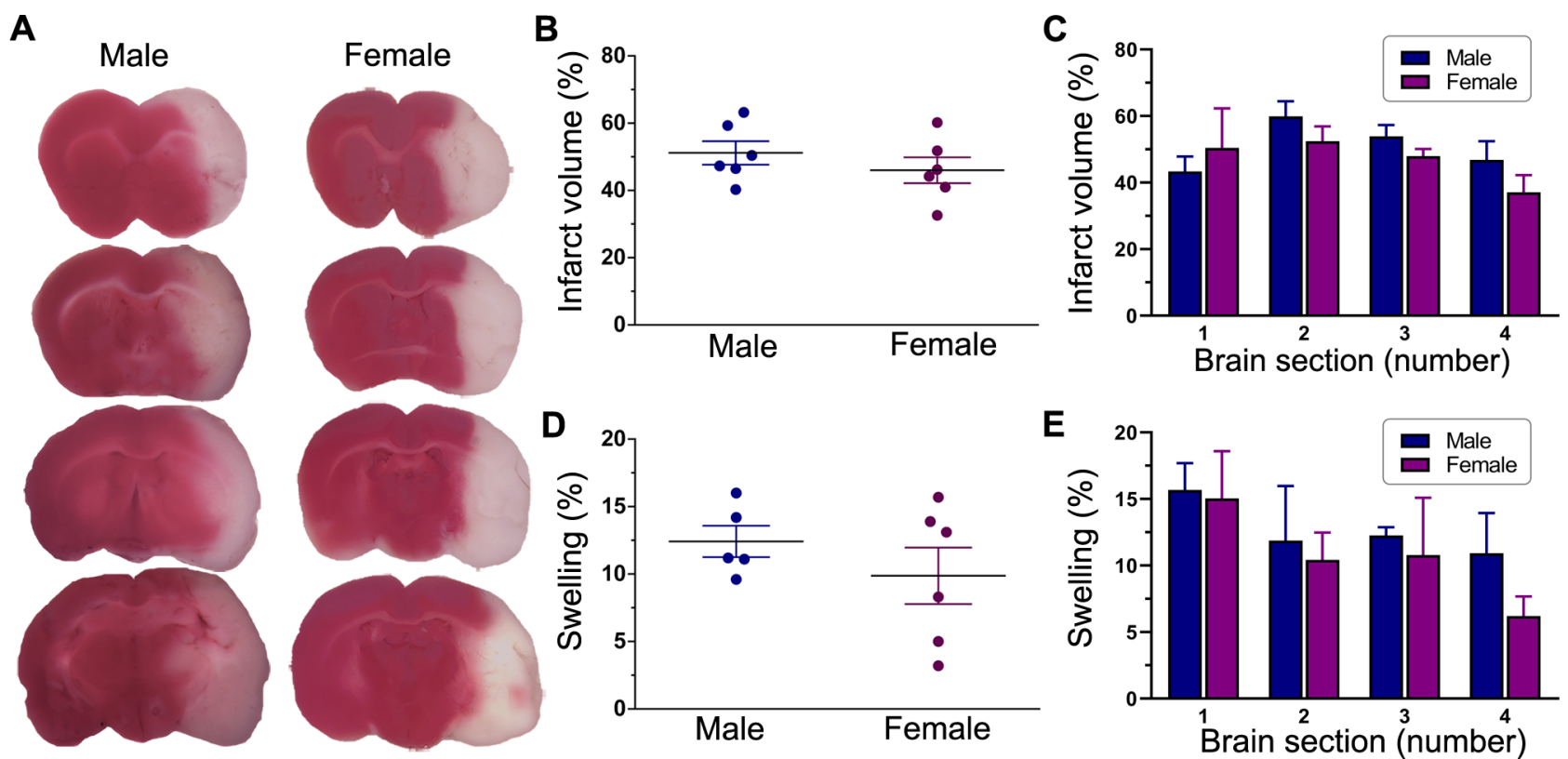

Figure 2 Post stroke brain damage and swelling. (A) Representative images of TTC-stained serial coronal brain sections from untreated male and female ischaemic rats. Animals were subjected to a 2-hour ischaemia and were subsequently euthanised 1 day after reperfusion. Sections are shown anterior to posterior (top to bottom) and span most of the forebrain from approximately $+2 \mathrm{~mm}$ to $-4 \mathrm{~mm}$ from bregma. The red-stained areas indicate normal healthy tissue, whereas the unstained (white) areas ipsilateral to MCAO indicate infarcted tissue. The column scatter plots show the total infarct volume (B) and swelling (D) at 1 day after reperfusion in untreated male and female ischaemic rats. Values are expressed as mean \pm SEM. Group N's were six males and six females for infarct volume and five males and six females for swelling. No statistically significant differences were found between the two groups in either infarct volume or brain swelling ( $p>0.05$, unpaired $t$-test). The bar graphs show the section-by-section analysis of TTC staining in untreated male and female ischaemic rats for infarct volume (C) and swelling (E). Values are expressed as mean+SEM (group N's as above). Two-way repeated measures analysis of variance did not reveal any significant differences between the two groups in either infarct volume or swelling. MCAO, middle cerebral artery occlusion; TTC, triphenyltetrazolium chloride.

of ipsilateral hemisphere-volume of contralateral hemisphere)/volume of contralateral hemisphere] $\times 100$.

Rats treated with MSC or vehicle were subsequently subjected to a battery of standard neurological/neurobehavioral tests, including the Modified Neurological Severity Score assessment (mNSS), ${ }^{29}$ adhesive tape removal test, ${ }^{30}$ beam walk test ${ }^{31}$ and accelerating rotarod. The tests were administered at regular intervals (at days $1,3,5,7$ and 14) after reperfusion to determine the time course of neurological and functional recovery after stroke. The detailed methodology including data collection, exclusion criteria and statistical analysis is provided in online supplemental material.

\section{RESULTS}

Infarct volume and extent of swelling are similar in both males and females

TTC staining of thick coronal brain sections was used to delineate infarcted from normal tissue as shown in figure $2 \mathrm{~A}$. The estimated total infarct volume and extent of swelling (both expressed as a percentage of the total contralateral hemisphere volume) were $51.2 \% \pm 3.8 \%$ and $12.4 \% \pm 1.2 \%$, respectively, in males and $46.0 \% \pm 3.2 \%$ and $9.9 \% \pm 2.3 \%$, respectively, in females at 1 day after reperfusion (figure 2B,D). The mean infarct volumes and extents of swelling were not statistically different in males and females $(\mathrm{p}=0.343$ and $\mathrm{p}=0.341$, respectively). A more detailed analysis comparing each respective brain section along the anterior-posterior axis also did not find any significant sex-related differences in either infarct size $(\mathrm{p}=0.353)$ or swelling $(\mathrm{p}=0.871)$ (figure 2C,E). Overall, these results suggest that the degree of post stroke brain damage (ie, pathological lesions of stroke) before treatment was similar in both males and females.

\section{Post stroke neurological scores are higher in males than} females and MSC treatment reduces the scores in both sexes The present results were obtained on 48 of the original 80 male and female rats allocated to this part of the study. Following cerebral ischaemia, neurological assessment of stroke as rated by the mNSS revealed scores of $12.9 \pm 0.3$ for the males $(n=25)$ and $10.4 \pm 0.4$ for the females $(n=23)$. These values were obtained 1 day after reperfusion (prior to treatment randomisation) and compared with a pre ischaemic baseline score of 0.0 for both sexes. The neurological scores of the females were significantly lower $(\mathrm{p}<0.0001)$ than the males and correspond to a mild stroke in the female rats versus a severe stroke in the male rats. Based on the neurological scores (and other test results described below), there appears to be 


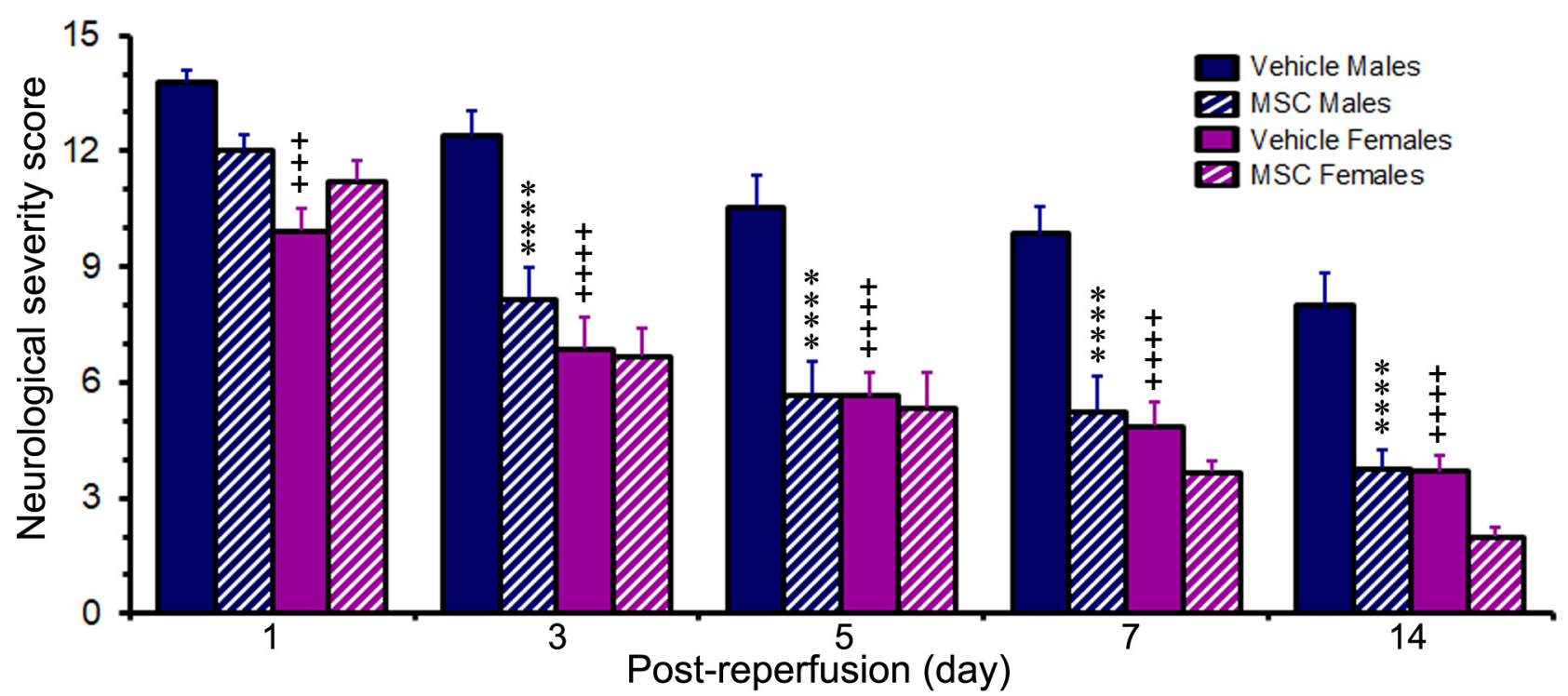

Figure 3 Post stroke changes in Modified Neurological Severity Score. The bar graph shows the neurological scores of rats subjected to a 2-hour ischaemia and subsequently treated with MSC or vehicle control 1 day after reperfusion. Values are expressed as mean+SEM. Group N's were 13 for the Vehicle Male group, 14 for the Vehicle Female group, 12 for the MSC Male group and 9 for the MSC Female group except for the 14-day timepoint where the group N's were 10 (Vehicle Males), 13 (Vehicle Females), 8 (MSC Males) and 7 (MSC Females). Data were analysed by two-way repeated measures ANOVA followed by Tukey's multiple comparisons test (for the 1-7-day timepoints) or by two-way ANOVA followed by Sidak's post hoc test (for the 14-day timepoint). ${ }^{* * * *} p<0.0001$ versus the same-sex vehicle group. ${ }^{+++} p<0.001 ;{ }^{+++} p<0.0001$ versus males receiving the same treatment. ANOVA, analysis of variance; MSC, mesenchymal stem cells.

a sex difference in the clinical manifestations of stroke with females generally less affected than males despite showing similar pathological lesions of stroke (ie, infarct volume and swelling).

Following randomisation of animals to the treatment groups, the neurological breakdown scores were $13.8 \pm 0.3$ and $9.9 \pm 0.6$ for the vehicle-treated male and female rats, respectively, and $12.0 \pm 0.4$ and $11.2 \pm 0.6$ for the MSC-treated male and female rats, respectively. The scores of the two vehicle-treated cohorts were significantly different $(p<0.001)$ from each other, while the scores of the two MSC-treated cohorts were not (figure 3). Thus, the randomisation of the male and female rats to the respective treatments (ie, vehicle control vs MSC) was effective, as no significant sex-dependent differences were observed between the control and treated groups at 1 day after reperfusion. The neurological scores of the rats in each cohort gradually declined over the course of the study as the animals recovered from the stroke. At the end of the study, the severity of the strokes was relatively mild in both the MSC-treated groups and the vehicletreated females but remained moderately severe in the vehicle-treated males.

In the vehicle-treated cohorts, the neurological scores of the females were significantly lower than the scores of the males at all the timepoints studied $(\mathrm{p}<0.001$ at day 1 and $\mathrm{p}<0.0001$ at days 3, 5, 7 and 14). By contrast, in the MSC-treated cohorts, the neurological scores of the females and the males were not significantly different at any timepoint.
In males, the neurological scores were significantly lower $(\mathrm{p}<0.0001)$ in the MSC-treated group compared with the vehicle-treated group at days 3, 5, 7 and 14 . Although the neurological scores tended to be lower in the MSC-treated female group compared with the vehicletreated group, the decreases were not statistically significant at any of the timepoints. At the end of the study (day 14 after reperfusion), the elevated neurological scores of the males were decreased to $8.0 \pm 0.8$ in the vehicle-treated group and to $3.8 \pm 0.5$ in the MSC-treated group. In the females, the elevated neurological scores were decreased to $3.7 \pm 0.4$ in the vehicle-treated group and $2.0 \pm 0.2$ in the MSC-treated group. Thus, MSC treatment appeared to have a greater impact on reducing the neurological stroke severity in males than in females.

\section{MSC treatment facilitates the recovery of somatosensory function in both sexes}

Prior to ischaemia induction, the baseline sticky-tape ratio of all the rats was approximately 1 , indicating little or no difference between the two forelimbs. Following MCAO however, the sticky-tape ratio fell below 1 (and often to zero) depending on the severity of the strokeinduced somatosensory deficit. At 1 day after reperfusion, the sticky-tape ratios were $0.00 \pm 0.00$ and $0.18 \pm 0.08$ for the vehicle-treated male and female rats, respectively, and $0.20 \pm 0.09$ and $0.08 \pm 0.04$ for the MSC-treated male and female rats, respectively. These low ratios collectively indicate that somatosensory function was severely compromised in all the cohorts at this early timepoint after 


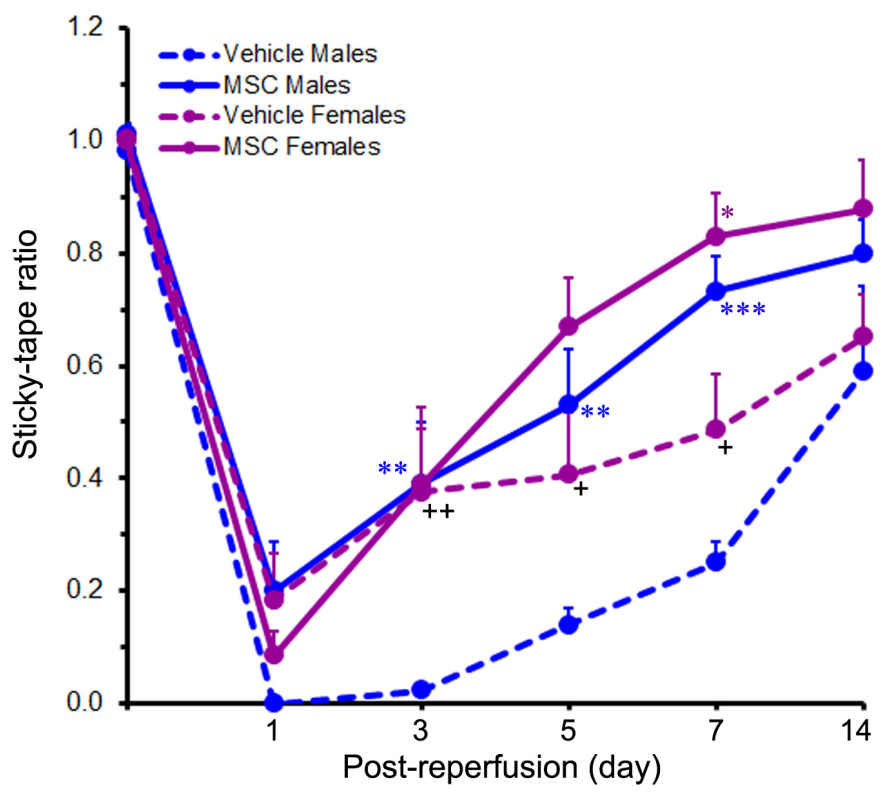

Figure 4 Treatment with MSC promotes the recovery of somatosensory function. The line graph shows the stickytape ratios of rats subjected to a 2-hour ischaemia and subsequently treated with MSC or vehicle control 1 day after reperfusion. Values are expressed as mean+SEM. Group N's were 13 for the Vehicle Male group, 14 for the Vehicle Female group, 12 for the MSC Male group and 9 for the MSC Female group except for the 14-day timepoint where the N's were 7 (Vehicle Males), 10 (Vehicle Females), 8 (MSC Males) and 7 (MSC Females). Data were analysed by two-way repeated measures ANOVA followed by Tukey's multiple comparisons test (for the 1-7-day timepoints) or two-way ANOVA followed by Sidak's post hoc test (for the 14-day timepoint). ${ }^{*} \mathrm{p}<0.05$; ${ }^{* \star} \mathrm{p}<0.01 ;{ }^{\star \star \star} \mathrm{p}<0.001$ versus the same-sex vehicle group. ${ }^{+} p<0.05 ;{ }^{++} p<0.01$ versus males receiving the same treatment. ANOVA, analysis of variance; MSC, mesenchymal stem cells.

reperfusion (figure 4). Furthermore, no significant sexrelated differences were observed $(\mathrm{p}=0.476)$. The stickytape ratios of the rats in each cohort gradually increased towards baseline levels over the course of the study until 14 days after reperfusion.

The sticky-tape ratios of the females compared with those of the males were significantly higher in the vehicle-treated cohorts (indicating less impairment), at days $3(\mathrm{p}<0.01), 5(\mathrm{p}<0.05)$ and $7(\mathrm{p}<0.05)$, but were not significantly different in the MSC-treated cohorts at any timepoint studied. These results suggest that the females initially recovered faster than the males in the vehicle control cohorts but recovered similarly to the males in the treatment cohorts.

In males, the sticky-tape ratios of the MSC-treated group were significantly higher than those of the vehicle-treated group at days $3(\mathrm{p}<0.01), 5(\mathrm{p}<0.01)$ and $7(\mathrm{p}<0.001)$, indicating a persistent treatment effect. In females however, the sticky-tape ratio was significantly higher in the MSCtreated group versus the vehicle-treated group only at day $7(\mathrm{p}<0.05)$, suggesting a diminished treatment effect. At the end of the study (day 14 after reperfusion), the decreased sticky-tape ratios of the males were increased to $0.59 \pm 0.15$ in the vehicle-treated group and to $0.80 \pm 0.06$ in the MSC-treated group. Similarly, the decreased stickytape ratios of the females were increased to $0.65 \pm 0.08$ in the vehicle-treated group and to $0.88 \pm 0.09$ in the MSCtreated group. Although the sticky-tape ratios were markedly increased in all the cohorts at day 14 relative to day 1 , the observed differences between and within treatments were not sex dependent.

\section{MSC treatment promotes the recovery of coordinated motor movement in both males and females}

The beam walk score of rats provides an overall measure of the animal's motor coordination/integration and balance (with 6 being a normal score). Following cerebral ischaemia, beam walk performance drastically declined in both male and female rats with most of the animals unable to stay on the beam ( $($ core $=0$ ) or unable to move $($ score $=1)$. At 1 day after reperfusion, the beam walk scores across the different treatments were $0.1 \pm 0.1$ (for the vehicle-treated males), $1.2 \pm 0.4$ (for the vehicle-treated females), $0.9 \pm 0.5$ (for the MSC-treated males) and $0.4 \pm 0.4$ (for the MSC-treated females). These scores indicate severe impairment of motor function in all the cohorts of animals (figure 5A). The degree of motor disruption was not related to sex $(\mathrm{p}=0.327) 1$ day after reperfusion. The beam walk scores of the rats in each cohort progressively increased over the course of the study until 14 days after reperfusion.

In the vehicle-treated cohorts, the beam walk scores of the females were significantly higher than the scores of the males (indicating less impairment) at days $3(\mathrm{p}<0.01)$, $5(\mathrm{p}<0.05), 7(\mathrm{p}<0.05)$ and $14(\mathrm{p}<0.05)$. These results suggest that the female rats showed faster recovery from stroke than the male rats. In the MSC-treated cohorts, the beam walk scores were significantly elevated in the females compared with the males at days $7(\mathrm{p}<0.01)$ and $14(\mathrm{p}<0.05)$. At these timepoints, the beam walk scores of the MSC-treated females returned to approximately baseline levels, but were still below baseline in the MSCtreated males.

In males, the beam walk scores of the MSC-treated group were significantly higher than the scores of the vehicle-treated group at days $3(\mathrm{p}<0.05), 5(\mathrm{p}<0.01)$, $7(\mathrm{p}<0.05)$ and $14(\mathrm{p}<0.001)$, indicating a persistent treatment effect. In females, the beam walk scores were significantly higher in the MSC-treated group compared with the vehicle-treated group at days $7(p<0.001)$ and $14(\mathrm{p}<0.001)$, indicating a clear treatment effect. At the end of the study, the decreased beam walk scores in males were increased to $1.8 \pm 0.4$ in the vehicletreated group and to $4.4 \pm 0.6$ in the MSC-treated group. Similarly, the decreased beam walk scores in females increased to $3.4 \pm 0.5$ in the vehicle-treated group and to $6.0 \pm 0.0$ in the MSC-treated group, indicating full recovery. 

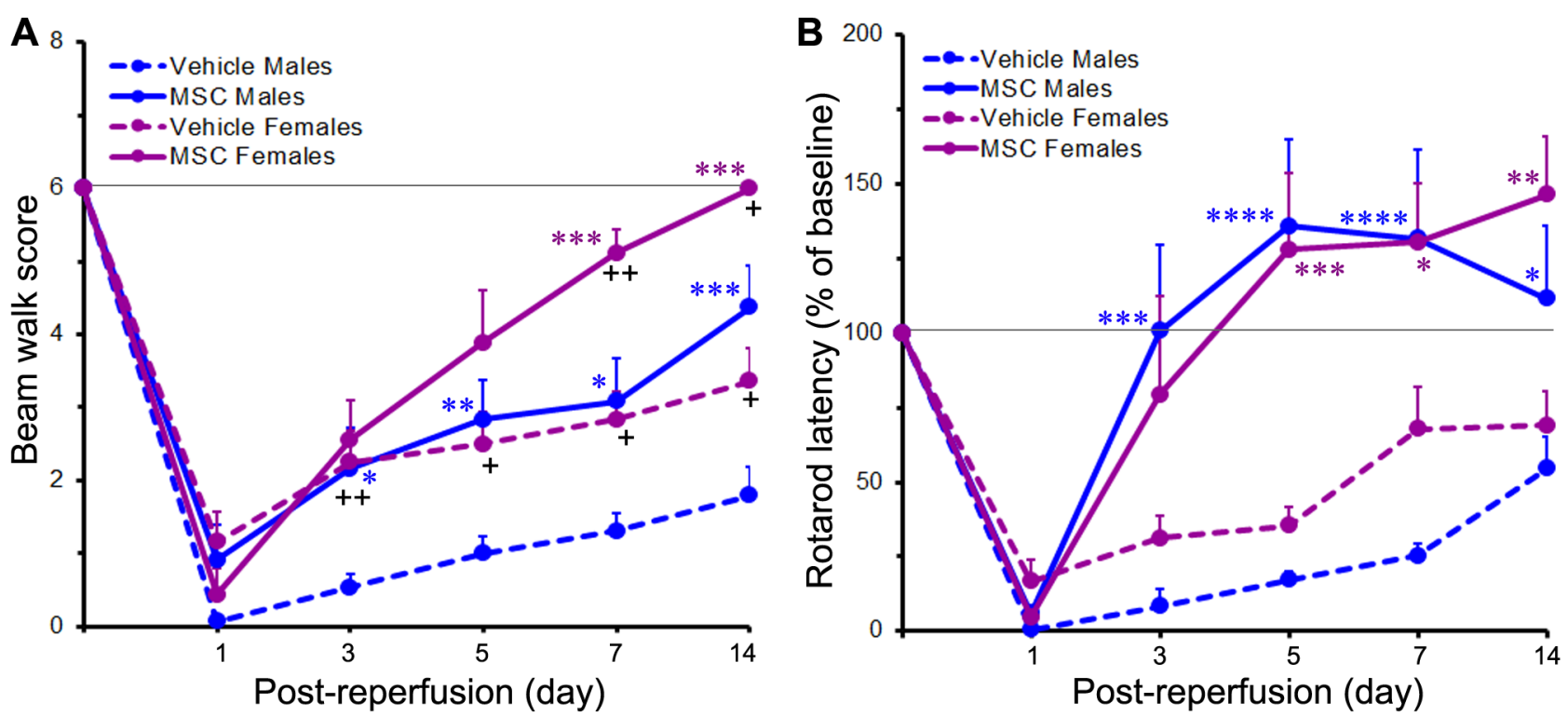

Figure 5 MSC treatment improves post stroke beam walk scores and rotarod performance. The line graphs show the beam walk scores (A) or rotarod latencies (expressed as a percent of baseline) (B) of rats subjected to a 2-hour ischaemia and subsequently treated with MSC or vehicle control 1 day after reperfusion. Values are expressed as mean+SEM. For the beam walk test, group N's were 13 for the Vehicle Male group, 14 for the Vehicle Female group, 12 for the MSC Male group and 9 for the MSC Female group except for the 14-day timepoint where the N's were 10 (Vehicle Males), 13 (Vehicle Females), 8 (MSC Males) and 7 (MSC Females). For the rotarod test, group N's were 10 for the Vehicle Male group, 14 for the Vehicle Female group, 10 for the MSC Male group and 9 for the MSC Female group except for the 14-day timepoint where the N's were 8 (Vehicle Males), 13 (Vehicle Females), 8 (MSC Males) and 7 (MSC Females). The horizontal line in each graph indicates the pre ischaemic baseline level. Data were analysed by two-way repeated measures ANOVA followed by Tukey's multiple comparisons test (for the 1-7-day timepoints) or by two-way ANOVA followed by Sidak's post hoc test (for the 14-day timepoint). ${ }^{*} \mathrm{p}<0.05$; ${ }^{* \star} p<0.01 ;{ }^{* \star *} p<0.001 ;{ }^{* \star * *} p<0.0001$ versus the same-sex vehicle group. ${ }^{+} p<0.05 ;{ }^{++} p<0.01$ versus males receiving the same treatment. ANOVA, analysis of variance; MSC, mesenchymal stem cells.

\section{MSC treatment promotes the recovery of locomotor performance in both sexes}

The accelerating rotarod performance of rats provides a quantitative measure of motor function.

Prior to ischaemia induction, the baseline rotarod latencies (in seconds) for the male and female rats were 72.3 $\pm 4.4(\mathrm{n}=20)$ and 69.3 $\pm 3.6(\mathrm{n}=23)$, respectively, and were not significantly different $(\mathrm{p}=0.599)$. Following cerebral ischaemia, there was a marked reduction in the ability of the animals to walk on the rotarod. At 1 day after reperfusion, the rotarod latencies (expressed as a percentage of baseline values) were $0.6 \% \pm 0.4 \%$ and $16.8 \% \pm 7.0 \%$ for the vehicle-treated male and female rats, respectively, and $5.8 \% \pm 2.2 \%$ and $4.8 \% \pm 2.0 \%$ for the MSCtreated male and female rats, respectively. The decreases in rotarod latencies were highly significant $(p<0.0001)$ across all treatment groups and indicate severe impairment of motor function in all the stroke-induced animals (figure 5B). The rotarod latencies quickly (within 5 days after reperfusion) returned to baseline levels or above in the MSC-treated male and female cohorts. However, they only gradually increased in the vehicle-treated male and female cohorts and were still below baseline levels 14 days after reperfusion. The rotarod latencies of the females and the males in both the MSC-treated cohorts and the vehicle-treated cohorts were not significantly different from each other at any of the timepoints studied.
In males, the rotarod latencies were significantly higher in the MSC-treated group compared with the vehicle-treated group at days $3(\mathrm{p}<0.001), 5(\mathrm{p}<0.0001), 7$ $(p<0.0001)$ and $14(p<0.05)$. The results in females were similar to those in males. Thus, in females, the rotarod latencies were significantly higher in the MSC-treated group compared with the vehicle-treated group at days 5 $(\mathrm{p}<0.001), 7(\mathrm{p}<0.05)$ and $14(\mathrm{p}<0.01)$, but not at day 3 .

At the end of the study (day 14 after reperfusion), the rotarod latencies in males were $54.5 \% \pm 11.0 \%$ in the vehicle-treated group and $111.7 \% \pm 24.3 \%$ in the MSCtreated group. Similarly, the rotarod latencies in females were $69.2 \% \pm 11.5 \%$ in the vehicle-treated group and $146.3 \% \pm 19.9 \%$ in the MSC-treated group. Overall, these data clearly show that MSC treatment improved rotarod performance in male and female rats to a generally similar degree. Finally, we observed no significant sexrelated differences either within a particular treatment or between treatments at any of the timepoints studied.

\section{DISCUSSION}

In this study, employing a battery of neurobehavioral tests and multiple timepoint assessments, we showed that treatment with human umbilical cord blood-derived MSC 1 day after reperfusion was efficacious in both male and female stroke-induced rats. Despite significant sex-dependent 
post ischaemic sensory and motor deficits, MSC treatment significantly improved the neurological recovery in both males and females.

The MSC used in this study originated primarily from an allogenic source are immature and elicit a lower incidence of graft rejection, graft versus host disease and post-transplant infections. ${ }^{32} 33$ Therefore, we did not administer any immunosuppressant drugs to MSC-treated rats. Furthermore, because there is a strong involvement of an immune component in the pathology of ischaemic stroke, the use of immunosuppressant drugs in stroke research studies would complicate the interpretation of results. Treatment of stroke-induced rats with MSC (at exactly the same dose, route and time after reperfusion as used herein) was previously shown to reduce apoptosis and post stroke brain damage, downregulate DNA damage-inducing genes, upregulate DNA repair genes and attenuate the induction of matrix metalloproteinases in the ischaemic brain. ${ }^{12-14}$ Furthermore, the administration of MSC within a few minutes to up to 3 days after ischaemia at much larger doses was shown earlier to attenuate infarct volume and suppress inflammatory responses as well as neuronal apoptosis in preclinical rodent stroke models. ${ }^{15} 171834$

Differentiation and paracrine signalling have been implicated as mechanisms by which stem cells promote tissue repair. ${ }^{35}$ Differentiation contributes to the therapeutic efficacy of stem cell treatment by regenerating damaged tissue. However, delivery of the administered cells into the ischaemic brain is believed to be crucial for successful treatment. The MSC administered to strokeinduced animals may reach the ischaemic brain via the damaged blood-brain barrier (BBB). Early disruption of the BBB begins within the first 3 hours after MCAO. ${ }^{36}$ The second peak of BBB disruption occurs between 24 hours and 72 hours, as in the transient occlusion model used in this study. ${ }^{37}$ Thus, MSC treatment that is initiated too early (within a day after reperfusion) or too late (3 or more days after reperfusion) may not help recovery due to the limited access of MSC to the brain. The timing of MSC treatment is therefore more critical than the actual dose. Our recent studies have demonstrated the presence of fluorescently labelled MSC in both cortical and striatal regions of the ischaemic brain following their administration to stroke-induced rats, using the same dosing and timing parameters employed in the current study. ${ }^{13}$ Reduced infarct volumes and improved behavioural functions have also been reported despite the absence of administered cells in the ischaemic brain. ${ }^{29} 3839$ The positive outcomes in these studies may be attributed to the paracrine signalling accomplished by the entry of therapeutic molecules secreted by the administered cells into the ischaemic brain. This notion is further supported by our recent study that showed a better post stroke neurological recovery of rats treated with exosomes secreted by MSC. ${ }^{16}$

In the present study, we tried to induce the same degree of stroke in both males and females (as judged by cerebral infarct measurements and neurological severity scores 1 day after reperfusion) by using appropriate size monofilaments and identical durations of ischaemia. The total infarct volume and extent of swelling of the ischaemic brain were found to be similar in both males and females. A more detailed, section-by-section analysis of the ischaemic brains also did not uncover any significant differences between males and females. However, as evidenced by the clinical manifestation of the stroke lesions (ie, neurological scores), MCAO resulted in severe stroke in males and more moderate stroke in females, suggesting the involvement of some factors unrelated to the pathological lesions in females. As highlighted in the introduction, oestrogens (and possibly other biological factors) in females could have played a role in reducing the severity of the clinical manifestations of the stroke lesions as observed in the present study when the same ischaemic duration was applied to both male and female rats. ${ }^{21-23}$

Following MSC treatment, the recovery of neurological function (as assessed by the mNSS) was substantial in males even though they exhibited more severe stroke symptoms. Although the neurological scores of the females were also reduced after MSC treatment, these reductions were not statistically different from vehicle treatment at any of the timepoints studied. When compared with the MSC-treated males, the neurological scores of the MSCtreated females were consistently lower throughout the study, especially at the latter two timepoints (ie, at days 7 and 14 after reperfusion). This could be attributed to the lower initial neurological scores and moderate stroke symptoms observed in females relative to males. In addition to mNSS assessments, we carried out more detailed analyses of post stroke sensory and motor deficits using several neurobehavioral tests such as the sticky-tape test, beam walk and rotarod. The results from all these tests showed a significant and dramatic recovery of sensory and motor function after MSC treatment in both males and females when compared with their respective vehicle control groups.

All test results except for the rotarod also revealed a significantly greater impairment of sensory and motor function in the vehicle-treated males versus females consistent with the neurological (mNSS) assessment data. Although a full or nearly full recovery of sensory and motor function was evident in both males and females after MSC treatment, the males exhibited the largest recovery gains despite showing more pronounced deficits in the various tests. In the beam walk test, MSC-treated females showed a significantly higher recovery level than the males at day 14 after reperfusion. Interestingly, in the rotarod test, full recovery of motor performance was observed in both sexes.

We believe that most, if not all, of the observed differences in treatment outcomes between the sexes may be attributed to differences in the initial severity of the induced strokes (males more affected than females) and not to actual differences in the effectiveness of the treatment per se in males versus females. Thus, while there is 
a clear sex difference in stroke severity, the effects of MSC treatment do not appear to be sex dependent.

Many studies have shown a beneficial effect of stem cells on stroke. Few, if any, have carried out such a detailed analysis of the potential therapeutic effects of stem cell treatment as we have in the present study, employing a wide range of sensory and motor tests. In most of the previous studies, the analysis of functional recovery was limited to only a few tests and/or timepoints. Consequently, the time course and extent of recovery following stem cell treatment is lacking in those studies, but is clearly defined in ours. Furthermore, we have also documented the efficacy of stem cell therapy in both male and female stroke-induced animals, as recommended by the Stroke Treatment Academic Industry Roundtable (STAIR).

In summary, MSC treatment was found to promote the recovery of sensory and motor function in both male and female animals despite sex differences in the severity of the clinical manifestations of the stroke lesions. Our results suggest that human umbilical cord blood-derived MSC treatment may be a promising therapy to facilitate post stroke neurological recovery.

\section{Author affiliations}

${ }^{1}$ Cancer Biology and Pharmacology, College of Medicine, University of Illinois, Peoria, Illinois, USA

${ }^{2}$ Pharmaceutical and Biomedical Sciences, California Health Sciences University, Clovis, California, USA

${ }^{3}$ Neurological Surgery, University of Wisconsin-Madison, Madison, Wisconsin, USA ${ }^{4}$ Health Sciences Education, College of Medicine, University of Illinois, Peoria, Illinois, USA

${ }^{5}$ Neurology, College of Medicine, University of Illinois, Peoria, Illinois, USA

${ }^{6}$ Neurology, Barrow Neurological Institute, Phoenix, Arizona, USA

${ }^{7}$ Neurosurgery, College of Medicine, University of Illinois, Peoria, Illinois, USA

${ }^{8}$ OSF HealthCare Illinois Neurological Institute, Peoria, Illinois, USA

${ }^{9}$ Pediatrics, College of Medicine, University of Illinois, Peoria, Illinois, USA

Acknowledgements The authors would like to thank the William E. McElroy Charitable Foundation, the OSF HealthCare Illinois Neurological Institute and the National Institute of Neurological Disorders and Stroke of the National Institutes of Health (NIH) for the financial assistance. The authors gratefully acknowledge Dr Stephen Lasley, Associate Editor of Neurotoxicology, for kindly reviewing the manuscript. Also, the authors thank Erika Sung and Pavani Unnam for their assistance in manuscript format and review.

Contributors KKV, JDK, DMP and DZW were involved in the study's conception, design and execution. KRN standardised and performed neurological tests and was involved in the animals' care. $\mathrm{BC}$ performed animal surgeries and assisted KRN in data collection. KRN and BC contributed equally to the study. KKV, CF and SRC analysed the data. KKV drafted the manuscript. CF edited the manuscript. All authors reviewed the draft manuscript and read and approved the final manuscript.

Funding This work was supported by research grants from the William E. McElroy Charitable Foundation, the OSF HealthCare Illinois Neurological Institute and the National Institute of Neurological Disorders and Stroke of the National Institutes of Health (award number R01NS102573) to KKV. The funders had no role in the study design, data collection, analysis, data interpretation, the decision to publish or preparation of the manuscript. The content is solely the responsibility of the authors and does not necessarily represent the official views of the funding agencies.

Competing interests None declared.

Patient consent for publication Not required.

Ethics approval The Institutional Animal Care and Use Committee (IACUC) of the University of Illinois College of Medicine at Peoria approved all surgical interventions and preoperative and postoperative animal care. All the animal experiments conducted were in accordance with the IACUC approved animal protocol.

Provenance and peer review Not commissioned; externally peer reviewed.
Data availability statement All data relevant to the study are included in the article or uploaded as supplementary information. Data (raw data of the performed experiments and the quantified and analysed data, including the statistical analysis) are available on reasonable request.

Open access This is an open access article distributed in accordance with the Creative Commons Attribution Non Commercial (CC BY-NC 4.0) license, which permits others to distribute, remix, adapt, build upon this work non-commercially, and license their derivative works on different terms, provided the original work is properly cited, appropriate credit is given, any changes made indicated, and the use is non-commercial. See: http://creativecommons.org/licenses/by-nc/4.0/.

ORCID iD

Krishna Kumar Veeravalli http://orcid.org/0000-0002-2243-004X

\section{REFERENCES}

1 Kenmuir CL, Wechsler LR. Update on cell therapy for stroke. Stroke Vasc Neurol 2017;2:59-64.

2 Liu X, Ye R, Yan T, et al. Cell based therapies for ischemic stroke: from basic science to bedside. Prog Neurobiol 2014;115:92-115.

3 Boese AC, Le Q-SE, Pham D, et al. Neural stem cell therapy for subacute and chronic ischemic stroke. Stem Cell Res Ther 2018;9:154,018-0913-2.

4 Chen X, Li Y, Wang L, et al. Ischemic rat brain extracts induce human marrow stromal cell growth factor production. Neuropathology 2002;22:275-9.

5 Li WY, Choi YJ, Lee PH, et al. Mesenchymal stem cells for ischemic stroke: changes in effects after ex vivo culturing. Cell Transplant 2008;17:1045-59.

6 Kim Y-J, Park H-J, Lee G, et al. Neuroprotective effects of human mesenchymal stem cells on dopaminergic neurons through antiinflammatory action. Glia 2009;57:13-23.

7 Liu Z, Li Y, Zhang RL, et al. Bone marrow stromal cells promote skilled motor recovery and enhance contralesional axonal connections after ischemic stroke in adult mice. Stroke 2011;42:740-4

8 Lai RC, Chen TS, Lim SK. Mesenchymal stem cell exosome: a novel stem cell-based therapy for cardiovascular disease. Regen Med $2011 ; 6: 481-92$

9 Song M, Mohamad O, Gu X, et al. Restoration of intracortical and thalamocortical circuits after transplantation of bone marrow mesenchymal stem cells into the ischemic brain of mice. Cell Transplant 2013;22:2001-15.

10 Acosta SA, Tajiri N, Hoover J, et al. Intravenous bone marrow stem cell grafts preferentially migrate to spleen and abrogate chronic inflammation in stroke. Stroke 2015;46:2616-27.

11 Marei HE, Hasan A, Rizzi R, et al. Potential of stem cell-based therapy for ischemic stroke. Front Neurol 2018;9:34.

12 Chelluboina B, Klopfenstein JD, Pinson DM, et al. Stem cell treatment after cerebral ischemia regulates the gene expression of apoptotic molecules. Neurochem Res 2014:39:1511-21.

13 Chelluboina B, Nalamolu KR, Klopfenstein JD. Stem cell treatment after ischemic stroke alters the expression of DNA damage signaling molecules. Journal of Stem Cell Research \& Therapeutics 2016;1:281-8.

14 Chelluboina B, Nalamolu KR, Mendez GG, et al. Mesenchymal stem cell treatment prevents post-stroke dysregulation of matrix metalloproteinases and tissue inhibitors of metalloproteinases. Cell Physiol Biochem 2017;44:1360-9.

15 Zhu Y, Guan Y-ming, Huang H-li, et al. Human umbilical cord blood mesenchymal stem cell transplantation suppresses inflammatory responses and neuronal apoptosis during early stage of focal cerebral ischemia in rabbits. Acta Pharmacol Sin 2014;35:585-91.

16 Nalamolu KR, Venkatesh I, Mohandass A, et al. Exosomes secreted by the cocultures of normal and oxygen-glucose-deprived stem cells improve post-stroke outcome. Neuromolecular Med 2019;21:529-39.

17 Chung D-J, Choi C-B, Lee S-H, et al. Intraarterially delivered human umbilical cord blood-derived mesenchymal stem cells in canine cerebral ischemia. J Neurosci Res 2009;87:3554-67.

$18 \mathrm{Kim}$ ES, Ahn SY, Im GH, et al. Human umbilical cord blood-derived mesenchymal stem cell transplantation attenuates severe brain injury by permanent middle cerebral artery occlusion in newborn rats. Pediatr Res 2012;72:277-84.

19 Savitz SI, Baron J-C, Fisher M, et al. Stroke treatment academic industry roundtable $\mathrm{X}$ : brain cytoprotection therapies in the reperfusion era. Stroke 2019;50:1026-31.

$20 \mathrm{Kim}$ T, Chelluboina B, Chokkalla AK, et al. Age and sex differences in the pathophysiology of acute CNS injury. Neurochem Int 2019;127:22-8 
21 Alkayed NJ, Harukuni I, Kimes AS, et al. Gender-linked brain injury in experimental stroke. Stroke 1998;29:159-66. discussion 166 : ] [published Online First: Jan.

22 Vannucci SJ, Willing LB, Goto S, et al. Experimental stroke in the female diabetic, db/db, mouse. J Cereb Blood Flow Metab 2001;21:52-60.

23 Suzuki S, Gerhold LM, Böttner M, et al. Estradiol enhances neurogenesis following ischemic stroke through estrogen receptors alpha and beta. J Comp Neurol 2007;500:1064-75.

24 Toung TJ, Traystman RJ, Hurn PD. Estrogen-mediated neuroprotection after experimental stroke in male rats. Stroke 1998;29:1666-70.

25 Toung TK, Hurn PD, Traystman RJ, et al. Estrogen decreases infarct size after temporary focal ischemia in a genetic model of type 1 diabetes mellitus. Stroke 2000;31:2701-6.

26 Santizo RA, Xu HL, Ye S, et al. Loss of benefit from estrogen replacement therapy in diabetic ovariectomized female rats subjected to transient forebrain ischemia. Brain Res 2002;956:86-95

27 Carswell HV, Bingham D, Wallace K, et al. Differential effects of 17 beta-estradiol upon stroke damage in stroke prone and normotensive rats. J Cereb Blood Flow Metab 2004;24:298-304.

28 Gordon KB, Macrae IM, Carswell HVO. Effects of 17beta-oestradio on cerebral ischaemic damage and lipid peroxidation. Brain Res 2005;1036:155-62.

29 Chen J, Sanberg PR, Li Y, et al. Intravenous administration of human umbilical cord blood reduces behavioral deficits after stroke in rats. Stroke 2001;32:2682-8.

30 Komotar RJ, Kim GH, Sughrue ME, et al. Neurologic assessment of somatosensory dysfunction following an experimental rodent model of cerebral ischemia. Nat Protoc 2007;2:2345-7.
31 Puurunen K, Jolkkonen J, Sirviö J, et al. An alpha(2)-adrenergic antagonist, atipamezole, facilitates behavioral recovery after focal cerebral ischemia in rats. Neuropharmacology 2001;40:597-606.

32 Knutsen AP, Wall DA. Kinetics of T-cell development of umbilical cord blood transplantation in severe T-cell immunodeficiency disorders. J Allergy Clin Immunol 1999;103:823-32.

33 Tse W, Laughlin MJ. Umbilical cord blood transplantation: a new alternative option. Hematology Am Soc Hematol Educ Program 2005;2005:377-83.

$34 \mathrm{Lim}$ JY, Jeong $\mathrm{CH}$, Jun JA, et al. Therapeutic effects of human umbilical cord blood-derived mesenchymal stem cells after intrathecal administration by lumbar puncture in a rat model of cerebral ischemia. Stem Cell Res Ther 2011;2:38.

35 Hocking AM, Gibran NS. Mesenchymal stem cells: paracrine signaling and differentiation during cutaneous wound repair. Exp Cell Res 2010;316:2213-9.

36 Huang ZG, Xue D, Preston E, et al. Biphasic opening of the blood-brain barrier following transient focal ischemia: effects of hypothermia. Can J Neurol Sci 1999;26:298-304.

37 Barber PA, Hoyte L, Kirk D, et al. Early T1- and T2-weighted MRI signatures of transient and permanent middle cerebral artery occlusion in a murine stroke model studied at 9.4T. Neurosci Lett 2005;388:54-9.

38 Borlongan CV, Hadman M, Sanberg CD, et al. Central nervous system entry of peripherally injected umbilical cord blood cells is not required for neuroprotection in stroke. Stroke 2004;35:2385-9.

39 Iskander A, Knight RA, Zhang ZG, et al. Intravenous administration of human umbilical cord blood-derived AC133+ endothelia progenitor cells in rat stroke model reduces infarct volume: magnetic resonance imaging and histological findings. Stem Cells Trans/ Med 2013;2:703-14. 